\title{
Posterior circulation
} ischemic stroke with aberrant vertebral artery cervical entrance at the C4 vertebral level

\author{
Branko N. Huisa, MD \\ L. Michelle Mathes, MD
}

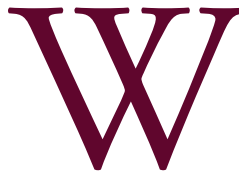

e describe 2 cases of recurrent ischemic stroke in the posterior circulation associated with an abnormal vertebral artery (VA) entrance into the transverse foramen. Neither patient had evidence of atherosclerotic disease, but both had arterial wall defects nearby the VA entrance at the C4 level suggestive of local arterial dissection.

\section{Case 1}

A 55-year-old man with nonvascular risk factors presented after sudden onset of unsteadiness and diplopia while riding his bike. His glucose, lipid profile, EKG, and hypercoagulability panel were within normal limits. His brain MRI showed multiple bilateral small restricted diffusion lesions in his cerebellum and left thalamus compatible with acute posterior circulation embolic strokes. Vascular imaging of his neck showed normal vessels with the exception of a right VA entering at the C4 level with nearby vessel wall defect (figure 1). Three months later, his symptoms recurred and a new left superior cerebellar artery ischemic lesion was confirmed by repeat brain MRI. He was switched from aspirin to Coumadin.

\section{Case 2}

A 57-year-old man presented with sudden onset of left-sided numbness and clumsiness that improved within 30 minutes. His brain MRI was negative for acute ischemia but he continued to have minor residual symptoms. He had had 2 previous posterior cerebral artery (PCA) ischemic strokes. At age 35 years, he suddenly lost his left visual field while working under his car. He was placed on aspirin. At age 50 years, he had another sudden episode of visual loss occurring after intense coughing affecting his contralateral visual field. He had recently been diagnosed with hypertension but had no other medical issues. His glucose, lipid profile, EKG, and hypercoagulability panel were within normal limits. His new brain MRI was negative for an acute infarct but showed previous ischemic lesions in both PCA territories and right cerebellum (figure 2) His magnetic resonance angiogram depicted an enlarged right VA segment at C4 level with a small arterial defect just before its entrance into the

Department of Neurology, University of New Mexico School of Medicine, Albuquerque.

Funding information and disclosures are provided at the end of the article. Full disclosure form information provided by the authors is available with the full text of this article at Neurology.org/cp.

Correspondence to: bhuisagarate@salud.unm.edu 
Figure $1 \quad$ Case 1

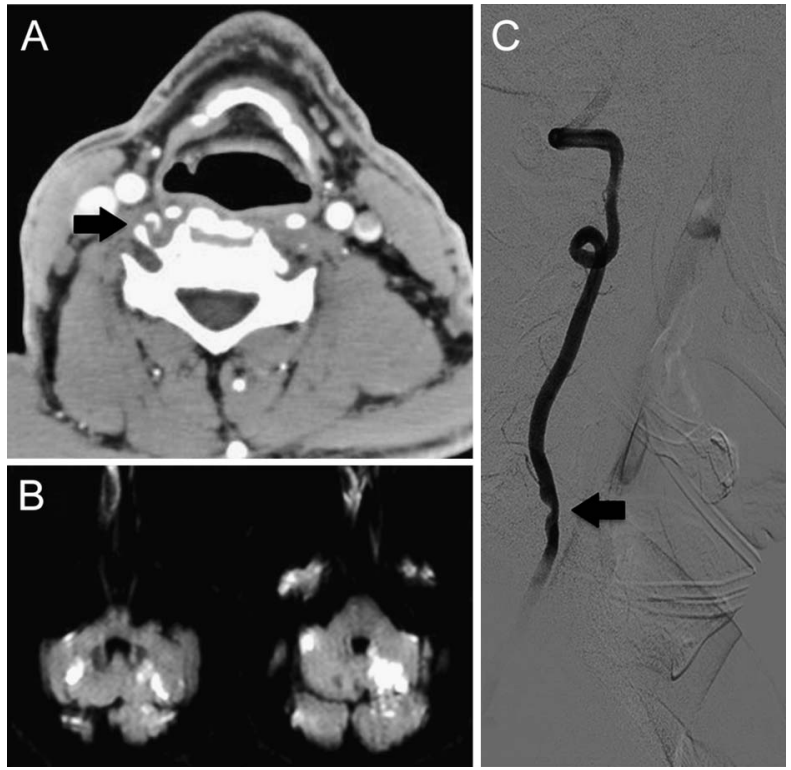

(A) CT angiogram of the neck shows the right vertebral artery (VA) near its entry at the C4 level. There is a crescentfilling defect in the VA wall (black arrow). (B) Brain MRI diffusion-weighted images show bilateral posterior circulation ischemic lesions. (C) Catheter angiogram of the right VA shows the arterial wall defect suggestive of a small arterial dissection (arrow).

vertebral canal. His neck CT angiography confirmed the findings (figure 2). We found no source of brain embolism after extensive cardiac, hematologic, and vascular studies.

\section{DISCUSSION}

The VA normally enters into the transverse foramen at level C6. The entry of the VA into the transverse foramen at the $\mathrm{C} 4$ level is rare and has been reported only in $1 \%$ of anatomical studies. ${ }^{1}$ The most likely stroke mechanism in our patients is local arterial dissection. This might have occurred by nearby osteophytes in the setting of rotation or hyperextension of the neck with subsequent injury of the arterial wall at the C4 entry level. Our PubMed search found only 2 previous cases with similar stroke mechanism, also in relationship with hyperextension and rotation of the patient's neck. ${ }^{2,3}$ Occlusion or partial occlusion of the VA has been described with neck motion (bow hunter's syndrome). ${ }^{4}$ In this condition, the dominant VA is commonly compressed at $\mathrm{C} 1-\mathrm{C} 2$ level and patients typically present with recurrent events of vertigo or fainting spells. ${ }^{5}$ We did not perform a dynamic cervical angiographic study with neck rotation in our patients to definitively rule out bow hunter's syndrome but the clinical presentation and imaging findings support an embolic event due to local dissection. Neither of our patients has evidence of atherosclerosis, cardioembolic source, coagulopathy, or connective tissue disease.

The recurrence of stroke after a VA dissection is low, with approximately $2.5 \%$ within the first 3 months. ${ }^{6}$ However, both cases had recurrence despite medical therapy, even years after the initial event. Recurrence might be related to reinjury of the VA intima followed by a new local dissection. Clinicians should be aware of this rare cause of stroke. Registries of extracranial arterial dissections will clarify the importance and frequency of this rare condition.

\section{REFERENCES}

1. Matula C, Trattnig S, Tschabitscher M, Day JD, Koos WT. The course of the prevertebral segment of the vertebral artery: anatomy and clinical significance. Surg Neurol 1997;48:125-131.

2. Fujiyama K, Motomura M, Shirabe S, et al. Locked-in syndrome and abnormal orientation of the right vertebral artery in a young man. Intern Med 1994;33:476-480. 


\section{Figure 2 Case 2}
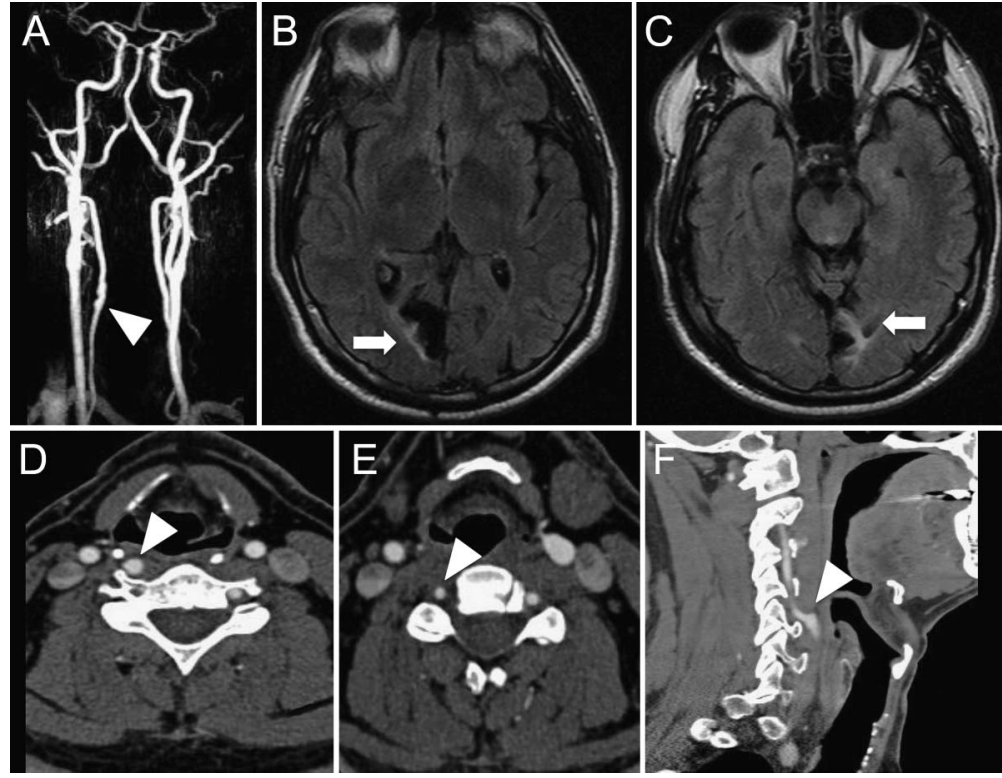

(A) Magnetic resonance angiogram of the neck shows a dilation of the right vertebral artery (VA) at the C4 level. There is a small artery defect at the same level (arrowhead). (B, C) Axial fluid-attenuated inversion recovery images show previous bilateral occipital strokes. (D) Axial CT angiogram images demonstrate a large right VA diameter at C4 level compared with (E) normal diameter at C3 level (arrowheads). (F) Neck CT angiography sagittal view shows the right VA enlargement at C4 with a wall defect immediately before its entry to the transverse foramen (arrowhead).

3. Jackson RS, Wheeler AH, Darden BV II. Vertebral artery anomaly with atraumatic dissection causing thromboembolic ischemia: a case report. Spine 2000;25:1989-1992.

4. Sorensen BF. Bow hunter's stroke. Neurosurgery 1978;2:259-261.

5. Choi KD, Choi JH, Kim JS, et al. Rotational vertebral artery occlusion: mechanisms and long-term outcome. Stroke 2013;44:1817-1824.

6. Debette S, Grond-Ginsbach C, Bodenant M, et al. Differential features of carotid and vertebral artery dissections: the CADISP study. Neurology 2011;77:1174-1181.

\section{STUDY FUNDING}

No targeted funding reported.

\section{DISCLOSURES}

The authors report no disclosures. Full disclosure form information provided by the authors is available with the full text of this article at Neurology.org/cp.

\section{What's New in Clinical Practice?}

Neurology ${ }^{\circledR}$ Clinical Practice now has podcasts available for download. The podcasts are introduced by Editor John R. Corboy, MD, FAAN, who highlights papers from the current issue. The interview that follows features authors discussing papers in more depth and bringing clinical implications to the fore. 